\title{
Avaliação da qualidade da prescrição da vacina anti-pneumocócica aos idosos
}

Marlene Sousa, * Luís Filipe Cavadas,* Rosa Branca Santos, * António Macedo*

\section{RESUMO}

Objectivos: Avaliar a qualidade da prescrição da vacina anti-pneumocócica (PPV) pelos Médicos de Família (MF), aos idosos inscritos no Centro de Saúde (CS) da Senhora da Hora.

Tipo de estudo: Avaliação da qualidade. Estudo retrospectivo.

Local: Centro de Saúde da Senhora da Hora, Matosinhos.

População: 10\% dos idosos inscritos nas listas de todos os MF do CS da Senhora da Hora, com idade $\geq$ a 65 anos a 31 de Dezembro de 2007.

Metodologia: Foi avaliada retrospectivamente (no período compreendido entre 1 de Janeiro de 2003 e 30 de Setembro de 2008) a qualidade técnico-científica da prescrição da PPV pelos $23 \mathrm{MF}$ do CS do estudo, numa amostra de $10 \%$ dos idosos inscritos nas listas de todos os MF, com idade $\geq$ a 65 anos a 31 de Dezembro de 2007, seleccionados aleatoriamente com reposição. Foram consultados dados do processo clínico e SAM ${ }^{\circledR}$. Utilizaram-se como padrão de qualidade para a prescrição os seguintes critérios: Muito Insuficiente se taxa de prescrição $<25 \%$, Insuficiente se taxa de prescrição $\geq 25 \%$ e $<50 \%$, Suficiente se taxa de prescrição $\geq 50 \%$ e $<75 \%$ e Bom se taxa de prescrição $\geq 75 \%$, visando uma intervenção educacional e mudanças estruturais. Resultados: Dos 418 processos avaliados, $238(56,9 \%)$ correspondiam a idosos do sexo feminino. A idade média foi de 75 anos (máximo de 98 anos e mínimo de 65 anos). Em 382 processos (91,4\%) não foi encontrado qualquer registo de prescrição da PPV estando esta presente em apenas $36(8,6 \%)$ processos, resultando num padrão de qualidade de prescrição muito insuficiente. Nos $23 \mathrm{MF}$ avaliados a taxa de prescrição variou entre $0 \%$ e 37,5\%. A faixa etária onde houve maior prescrição foi a dos 70 aos 74 anos.

Conclusão: O padrão de qualidade de prescrição da PPV no CS da Senhora da Hora foi muito insuficiente. A ausência de normas de orientação clínica portuguesas poderá estar na base desta baixa prescrição da vacina. Para melhorar o padrão de prescrição, propõem-se medidas estruturais (aviso informático para a vacinação, criação de circulares normativas) e educativas (reuniões de formação, palestras e folhetos informativos) dirigidas aos profissionais de saúde e idosos. Propõe-se reavaliação, após implementação das medidas correctoras iniciadas em Dezembro de 2008.

Palavras-Chave: Vacina anti-pneumocócica, Idosos, Registo da prescrição.

\section{INTRODUÇÃO}

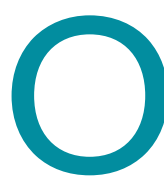

Streptococcus pneumoniae, um coco Gram positivo, com mais de 90 tipos capsulares descritos, é um dos agentes etiológicos mais frequentes de pneumonia, meningite e bacteriémia. ${ }^{1-4} \mathrm{~A}$ doença pneumocócica apresenta uma variação sazonal, com maior incidência nos meses de Inverno. ${ }^{1,2}$

A doença pneumocócica invasiva é uma causa $m a-$ jor de morbilidade e mortalidade. ${ }^{4,5}$ Afecta particular-

*Internos de Medicina Geral e Familiar do Centro de Saúde da Senhora da Hora Unidade Local de Saúde de Matosinhos mente as crianças muito jovens, os idosos e aqueles com imunodepressão ou doenças crónicas. ${ }^{1-5}$ Segundo a Organização Mundial de Saúde (OMS), na Europa e Estados Unidos da América (EUA), a doença invasiva sob a forma de bacteriémia afecta cada 15 a 19 por 100.000 idosos. ${ }^{4}$ As estatísticas do Center for Disease Control and Prevention (CDC) nos EUA referem cerca de 50.000 novos casos/ano de bacteriémia por pneumococos. ${ }^{6} \mathrm{~A}$ taxa de mortalidade atinge aproximadamente $20 \%$ e pode inclusive ser de $60 \%$ nos idosos. ${ }^{4-6}$ Actualmente existem cerca de $10 \%$ de indivíduos idosos no mundo (indivíduos com idade igual ou superior a 65 anos) e estima-se que em 2050 esta faixa etária re- 
presente $21 \%$ da população mundial. ${ }^{7}$

A vacinação anti-pneumocócica é considerada uma estratégia efectiva para diminuir as doenças associadas ao pneumococo, particularmente na população idosa..$^{1-6}$ O Departamento de Saúde e Serviços Humanos dos Estados Unidos da América, no seu relatório intitulado “The New Healthy People 2010", refere como objectivos nacionais atingir, em 2010, a vacinação de $90 \%$ dos adultos com idade igual ou superior a 65 anos. $\mathrm{O}$ mesmo documento refere uma taxa de vacinação de apenas $46 \%$ em $1998 .{ }^{8}$ Em Portugal estes dados não estão disponíveis.

Existem actualmente dois tipos de vacinas licenciadas na Europa que incluem um número variado de serótipos: a Vacina anti-pneumocócica polissacárida polivalente (PPV) - 23 serótipos; e a Vacina anti-pneumocócica sacárida conjugada adsorvida (PCV) - 7 serótipos. A vacina PPV protege contra a doença invasiva devida a 23 serótipos, em indivíduos com idade superior a 2 anos. A vacina PCV protege da doença causada por 7 serótipos, em indivíduos com idade inferior a 2 anos, protegendo igualmente contra manifestações de doença não invasiva. Ambas as vacinas são muito eficazes. $^{1,3,4,9}$

A vacina anti-pneumocócica PPV está instituída para os adultos com idade superior ou igual a 65 anos nos países da América do Norte e em muitos países Europeus. ${ }^{10,11}$ Entidades internacionais como a United States Preventive Services Task Force (USPSTF) e a Royal Australian College of General Practitioners (RACGP), incluem a vacinação anti-pneumocócica como uma actividade preventiva a realizar nesta população, sustentando as suas recomendações em evidência de boa qualidade. ${ }^{12,13}$ Segundo estas normas de orientação clínica, a vacina anti-pneumocócica PPV deve ser administrada a todos os indivíduos com idade igual ou superior a 65 anos, estando indicada para esta faixa etária uma toma única da vacina. Uma segunda dose é necessária para os idosos que tenham sido vacinados antes dos 65 anos e em que já tenha passado mais de 5 anos sobre esta primeira toma. ${ }^{1,12-14}$ Contudo a Direcção-Geral da Saúde ainda não contemplou o uso desta vacina nos idosos portugueses.

É objectivo deste estudo avaliar a qualidade da prescrição da PPV pelos Médicos de Família (MF) aos idosos inscritos no Centro de Saúde (CS) da Senhora da Hora.

\section{MÉTODOS}

\section{Dimensão estudada}

Qualidade técnico-científica da prescrição da vacina anti-pneumocócica aos idosos.

\section{Unidade de estudo}

Utentes: idosos inscritos nas listas de todos os médicos de Medicina Geral e Familiar (MGF) do Centro de Saúde da Senhora da Hora (23 profissionais), com idade igual ou superior a 65 anos a 31 de Dezembro de 2007. Excluíram-se os idosos sem Médico de Família.

Profissionais: todos os 23 médicos de MGF do Centro de Saúde da Senhora da Hora.

\section{Período Temporal}

Foram avaliados retrospectivamente os processos clínicos dos $23 \mathrm{MF}$ do CS em estudo, no período compreendido entre 1 de Janeiro de 2003 e 30 de Setembro de 2008 , relativos aos utentes com idade igual ou superior a 65 anos a 31 de Dezembro de 2007.

Tipo de Dados

De processo.

\section{Fonte de Dados}

Processos clínicos informatizados [versão 7.1 do Sistema de Apoio ao Médico (SAM)] e Processos clínicos manuscritos: Fichas Clínicas Individuais - Adultos (ULS-Mod.121.01) ou Folhas de Consulta - Adultos (ULS-Mod.13/99.017), ambas as fontes pesquisadas para cada utente.

Tipo de Avaliação

Interna.

\section{Critério}

O critério avaliado e o padrão de qualidade adoptado encontram-se descritos no Quadro I.

\section{Colheita de dados}

Foi efectuada pelos autores, entre 3 de Novembro e 15 de Novembro de 2008.

Avaliação temporal

Avaliação retrospectiva. 
QUADRO I. Critério avaliado e Padrão de qualidade adoptado

\section{Critério}

Registo da prescrição da vacina anti-pneumocócica no período de 01/01/2003 e 30/09/2008 em utentes com idade $\geq 65$ anos a 31 de Dezembro de 2007

\section{Amostra}

De base institucional. A partir do programa informático SINUS ${ }^{\circledast}$ foram listados todos os idosos inscritos nos $23 \mathrm{MF}$ com idade igual ou superior a 65 anos em $31 \mathrm{de}$ Dezembro de 2007. Destes, foram seleccionados $10 \%$ do total de idosos de forma aleatória sistemática com reposição, obtendo uma dimensão amostral de 418 utentes.

\section{Tipo de Intervenção}

Educacional e estrutural.

\section{Considerações éticas}

Este estudo cumpre todos os pressupostos ético-legais, tendo sido aprovado pela Comissão de Ética da ULS Matosinhos.

\section{RESULTADOS}

O estudo englobou a análise de 418 processos clínicos, dos quais 238 (56,9\%) correspondiam a utentes do sexo feminino. A idade média dos utentes foi de 75 anos para um máximo de 98 anos e um mínimo de 65 anos. No Quadro II está presente a caracterização da amostra estudada.

Em 382 processos (91,4\%) não foi encontrada qualquer prescrição da vacina anti-pneumocócica. A prescrição da vacina está presente em apenas 36 processos (taxa de prescrição média de 8,6\%; IC a 95\%; 5,9\%$-11,3 \%)$ o que se manifesta em um padrão de qualidade Muito Insuficiente.

Entre os 23 médicos prescritores avaliados a taxa de prescrição variou entre 0\% e 37,5\% (Figura 1).

Nos processos com prescrição da vacina anti-pneumocócica verificou-se que a faixa etária com maior

\begin{tabular}{|lc|}
\hline \multicolumn{2}{|l|}{ QUADRO II. Caracterização da amostra estudada } \\
\hline \multicolumn{2}{|l}{ Sexo } \\
$\quad$ Feminino \\
Masculino \\
\hline Idade & $238(56,9)$ \\
$65-69$ & $180(43,1)$ \\
$70-74$ & \\
$75-79$ & $112(26,8)$ \\
$80-84$ & $129(30,9)$ \\
$85-89$ & $73(17,5)$ \\
$\geq 90$ & $54(12,9)$ \\
\end{tabular}

prescrição foi a dos 70 aos 74 anos. O SAM ${ }^{\circledast}$ foi a fonte de dados usada em 23 (63,9\%) dos casos de prescrição enquanto o processo clínico manuscrito abrangeu 13 $(36,1 \%)$ casos. O ano em que houve mais prescrições da vacina foi 2007 com 18 (50\%) dos casos enquanto no ano de 2004 houve o menor número de prescrições com 1 caso $(2,8 \%)$.

\section{DISCUSSÃO}

A taxa de prescrição de vacina anti-pneumocócica foi de $8,6 \%$ (IC 95\%; 5,9\%-11,3\%) nos 418 processos avaliados, o que traduz um padrão muito insuficiente na prescrição desta vacina aos idosos do CS Senhora da Hora. A ausência de normas de orientação definidas em Portugal para a prescrição desta vacina poderá estar na origem de tão pequena taxa. Igualmente, a consulta de processos clínicos manuscritos poderá introduzir um viés de informação pela dificuldade de leitura de alguma da informação neles contida. Ainda assim os autores, prevendo que alguns dos MF utilizariam preferencialmente o processo manuscrito, optaram pela consulta aos mesmos de forma a abranger de modo mais fiável todas as prescrições da vacina. Contudo, a fonte preferencial de prescrição foi o processo informatizado. A análise do número de prescrições por ano sugere que as mesmas têm vindo a aumentar, o que pode traduzir uma maior sensibilização à prescrição da vacina por parte dos médicos que incluem esta atitude preventiva na sua prática clínica.

Como pontos fortes deste estudo, pode referir-se o recurso a um tamanho amostral e método de amostra- 


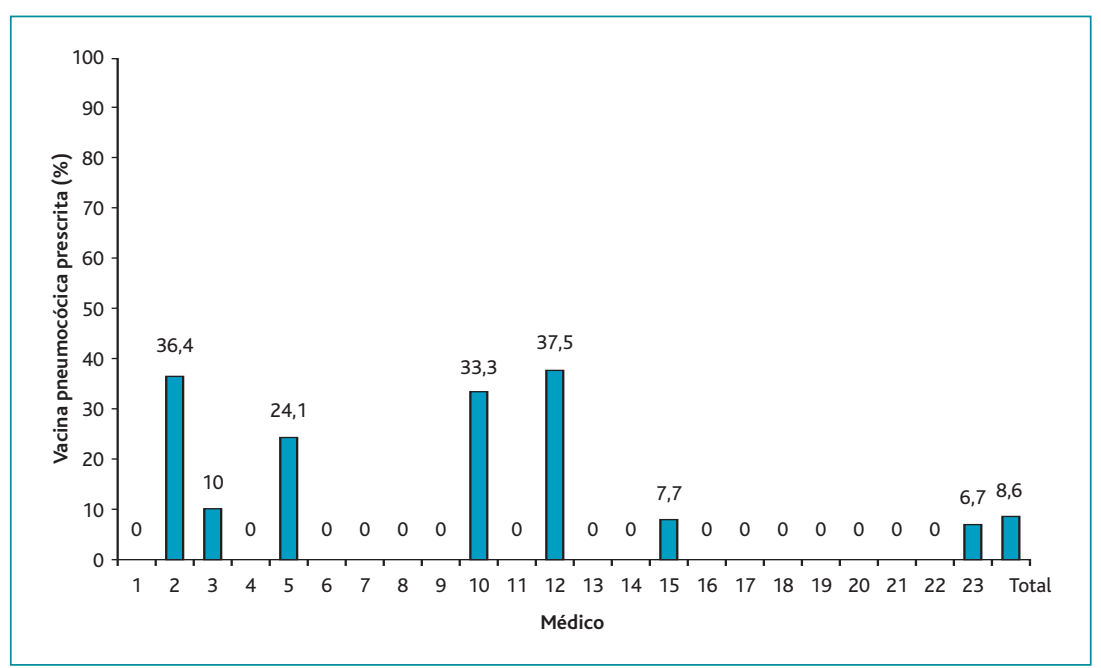

Figura 1. Taxa de prescrição da vacina anti-pneumocócica por Médico de Família.

gem adequados, permitindo obtenção de uma amostra de idosos representativa da população de idosos inscritos no CS da Senhora da Hora. O tema escolhido para o estudo é pertinente não só pela efectividade da vacina na diminuição da morbimortalidade associada à doença invasiva por pneumococos, mas também por se tratar de um tema pouco abordado em Portugal, apesar da existência de evidência de boa prática. Pode-se assim considerar este estudo como pioneiro nesta área.

Como aspectos negativos do estudo é de referir a utilização de dados não informatizados, associada ao viés já referido, bem como o período temporal adoptado que pode não ter considerado como prescrita uma vacina PPV efectuada em anos anteriores.

\section{PROPOSTAS DE INTERVENÇÃO}

Propõem-se intervenções a nível estrutural e educacional.

Está criado um grupo de trabalho destinado ao desenvolvimento contínuo de qualidade na prescrição da vacina anti-pneumocócica. Actualmente é constituído por médicos mas poderá posteriormente incluir outros grupos profissionais, nomeadamente enfermeiros e administrativos.

Este grupo iniciou a divulgação dos resultados deste trabalho em reunião de formação destinada aos profissionais médicos e irá organizar, de forma subsequente, novas reuniões sobre o tema, como forma de sensi- bilização dos restantes grupos profissionais do CS, motivando todos (médicos, enfermeiros e administrativos) a realizar um aconselhamento oportunista da vacinação anti-pneumocócica aos idosos que recorram ao CS por outros motivos (como é o caso dos pedidos de medicação crónica, realização de cuidados de enfermagem, entre outros). Nessas reuniões serão adoptadas estratégias inter-pares que visem colmatar deficiências desse aconselhamento.

Como formas de divulgação da prescrição desta vacina, irão ser distribuídos folhetos aos MF do CS explicando os benefícios da administração da vacina a este grupo etário. Serão, também, distribuídos folhetos aos utentes para que possam ficar informados acerca da existência e benefícios da vacina e serão organizadas palestras informativas e afixados posters incentivando esta vacinação.

Será proposta a colocação de um aviso informático no $\mathrm{SAM}^{\circledast}$ lembrando a ausência de prescrição de vacina para utentes com idade igual ou superior a 65 anos e sugerindo a vacinação.

Com estas acções o grupo pretende alertar a Direcção Geral da Saúde para a possível criação de Circulares Normativas e Informativas sobre a prescrição da vacina anti-pneumocócica e ajudar a promover um melhor registo da prescrição da vacina anti-pneumocócica, reflectindo-se na melhoria da prestação de cuidados ao idoso. 


\section{REFERÊNCIAS BIBLIOGRÁFICAS}

1. Salisbury D, Ramsay M, Noakes K, editors. Pneumococcal. In: Immunization against infectious disease: the Green Book. 3rd ed. London: Department of Health; 2006. p. 295-3311.

2. Hawley LA, Walker F, Whitney CG. Pneumococcal disease. In: VPD Surveillance Manual. 3rd ed. Washington, DC: Centers for Disease Control and Prevention; 2002. p. 9-14.

3. Atkinson W, Hamborsky J, McIntyre L, Wolfe S; Centers for Disease Control and Prevention. Epidemiology and prevention of vaccine-preventable diseases. 10th ed, 2nd printing. Washington, DC: Public Health Foundation; 2008. Disponível em: http://www.cdc.gov/vaccines/pubs/pinkbook/downloads/pneumo.pdf [acedido em 22/09/2008].

4. Pneumococcal vaccines:WHO position paper. Weekly Epidemiological Record 1999; 74: 177-83. Disponível em: http://www.who.int/wer/pdf/ 1999/wer7423.pdf [acedido em 22/09/2008].

5. Centers for Disease Control and Prevention. Prevention of pneumococcal disease: recommendations of the Advisory Committee on Immunization Practices (ACIP). MMWR 1997; 46 (No. RR-8).

6. Centers for Disease Control and Prevention. Health Information for International Travel 2008. Pneumococcal Disease. Atlanta, GA: US Department of Health and Human Services, Public Health Service; 2007.

7. Spalding MC, Sebesta SC. Geriatric screening and preventive care. Am Fam Physician 2008 Jul 15; 78 (2): 206-15.

8. U.S. Department of Health and Human Services. Healthy People 2010: Understanding and Improving Health. Washington, DC: U.S. Government Printing Office; 2008. Disponível em: http://www.healthypeople.gov/publications [acedido em 22/09/2008].

9. Centro Regional de Saúde Pública do Centro. Fichas de produto e segurança: vacinas não incluídas no Plano Nacional de Vacinação. Coimbra: ARS Centro; 2006.

10. Pebody RG, Leino T, Nohynek H, Hellenbrand W, Salmaso S, Ruutu P. Pneumococcal vaccination policy in Europe. Euro Surveill 2005 Sep; 10 (9): 174-8.
11. Gavazzi G, Wazieres B, Lejeune B, Rothan-Tondeur M. Influenza and pneumococcal vaccine coverages in geriatric health care settings in France. Gerontology 2007; 53 (6): 382-7.

12. The Guide to Clinical Preventive Services 2006: Recommendations of the U. S. Preventive Services Task Force (USPSTF). Disponível em: http://www.ahrq.gov/clinic/pocketgd.pdf [acedido em 22/09/2008].

13. The Royal Australian College of General Practitioners (RACGP). Guidelines for preventive activities in general practice. 6th ed. Disponível em: http://www.racgp.org.au/Content/NavigationMenu/ClinicalResources/RACGPGuidelines/TheRedBook/2005Redbook_6th_ed.pdf [acedido em 22/09/2008].

14. Galvão C. Actividades preventivas no idoso. Rev Port Clin Geral 2006 Nov-Dez; 22 (6): 731-7.

Os autores declararam não possuir conflitos de interesses

\section{AGRADECIMENTOS}

Aos Orientadores de Formação dos autores, pertencentes ao Grupo de Internos e Orientadores do Centro de Saúde da Senhora da Hora: Dr. ${ }^{a}$ Arminda Santos, Dr. ${ }^{a}$ Mónica Granja, Dr. ${ }^{a}$ Raquel Braga e Dr. ${ }^{\text {a }}$ Teresa Neto, pela colaboração na elaboração deste trabalho.

\section{ENDEREÇO PARA CORRESPONDÊNCIA}

Marlene Sousa

Centro de Saúde da Senhora da Hora

Rua da Lagoa, $s / n^{\circ}$

4460 Senhora da Hora

E-mail: marleneso@iol.pt

Recebido em 16/02/2009

Aceite para publicação em 15/07/2009 


\section{ABSTRACT}

\section{QUALITY EVALUATION OF THE PRESCRIPTION OF THE ANTI-PNEUMOCOCAL VACCINE IN THE ELDERLY}

Objectives: To evaluate the quality of prescription of anti-pneumococcal vaccine (APV) by Family Physicians (FP), to the elderly of the Primary Care Center (PCC) studied.

Type of study: Quality evaluation study. Retrospective study. Location: Primary Care Center of Senhora da Hora, Matosinhos

Population: $10 \%$ of elderly included in the lists of all the FP at the PCC of the study aged $\geq 65$ years at December 31 st, 2007 . Methods: We evaluated retrospectively (from January 1st, 2003 to September 30th, 2008) the technical and scientific quality of the prescription of APV by the 23 FP from the PCC of the study, in a sample of $10 \%$ of elderly aged $\geq 65$ years at December 31 st 2007 , selected randomly with replacement. We consulted the clinical data and SAM ${ }^{\circledR}$. The following criteria were used as quality standards for prescription: Very low quality if prescription rate was $<25 \%$, Low quality if prescription rate was $\geq 25 \%$ and $<50 \%$, Sufficient quality if prescription rate was $\geq 50 \%$ and $<75 \%$ and Good quality if prescription rate $\geq 75 \%$, seeking an educational intervention and structural changes.

Results: Of the 418 cases studied, 238 (56.9\%) accounted for elderly females. The average age was 75 years (maximum of 98 years and minimum of 65 years). In 382 cases (91.4\%) there was no registry of prescription of APV. The registry of prescription was present in only $36(8.6 \%)$ cases, resulting in a pattern of Very Low quality of prescription. In the 23 FP evaluated the rate of prescription varied between $0 \%$ and $37.5 \%$. The age group where there was the greatest prescription rate was 70 to 74 years. Conclusion: The quality of APV prescription in the study PCC was very low. The absence of appropriate Portuguese clinical guidelines may be in the basis of this low prescribing of the vaccine. To improve the rate of prescription the authors propose structural measures (computer warning information to the vaccination, establishment of specific guidelines) and education (training meetings, lectures and leaflets) directed to health professionals and elderly. The authors propose revaluation, after implementation of the corrective measures already initiated in December 2008.

Keywords: Anti-pneumococcal Vaccine; Elderly; Registry of Prescription. 